\title{
Design of photoacoustic microscope system based on labVIEW platform
}

\author{
Yang Bai, Chuncheng Zhang, Lvming Zeng and Guodong Liu* \\ Key Lab of Optic-Electronic and Communication, Jiangxi Sciences and Technology Normal University, \\ Nanchang 330038, China
}

Keywords: Virtual instrument, Digital oscilloscope, Photoacoustic imaging.

\begin{abstract}
A photoacoustic microscope system based on virtual instrument development environment is presented, including ultrasonic sensor, digital oscilloscope, laser diode, personal calculation and other hardware platforms. and developed supporting software and image reconstruction algorithms. In the subcutaneous angiography experiment, the distribution characteristics of the ear blood vessels in mouse were completely reproduced perfectly, and the spatial resolution of the system can reach $14 \mathrm{um}$. The system and method can potentially to develop into a non-invasive biological tissue structure and functional imaging technique.
\end{abstract}

\section{Introduction}

Pure optical imaging has the advantages of fast imaging, non-invasive, high resolution, etc. $[1,2]$, but with the increase of light penetration depth, the strong spatial scattering of light causes a rapid decrease in imaging spatial resolution; ultrasound imaging has the advantage of high-penetration type of tissue. It is easy to cause misdiagnosis because of imaging contrast is low for early lesions. The positron emission tomography has an important position in the current medical imaging research [3], but due to its complicated equipment, slow imaging speed, and existing dyeing. The development of the type of agent is limited, and its promotion has received restrictions; MRI has the advantages of less loss lessness and high resolution, and can obtain information such as tissue anatomy and physiological functions at the same time [4], but its sensitivity is low (micromolar level), and the cost is expensive, so researching and developing a low-cost, high-contrast and high-resolution non-destructive medical imaging method is a problem to be solved in the field of clinical medicine.

Photoacoustic imaging is a non-destructive bio-photonic medical imaging technology based on the difference in optical absorption between biological tissues and ultrasound [5-8]. It combines the advantages of high contrast of pure optical imaging and high penetration depth of pure blood imaging [9-10]. As one of the important branches of photoacoustic imaging, photoacoustic microscopy also has the above advantages, which can realize the

\footnotetext{
*Corresponding author: Alexbaiy@,163.com
} 
imaging image of millimeter-scale detection depth and micron-level imaging accuracy [11-13], but the existing photoacoustic microscopy system usually uses volume. A large solid-state laser or an OPO laser is used as an excitation source for the system and is constrained in practical applications [13-15]. In this paper, a "virtual instrument" (VI) is used to design a small-scale high-resolution photoacoustic microscopy imaging system. The excitation source uses laser diodes with small size, low price and high repetition rate [15-18]. It was initially applied to the imaging of subcutaneous vascular imaging in mouse ears.

\section{Hardware design}

The whole experimental system is mainly composed of PC, high-definition digital oscilloscope and 3D mobile platform. As shown in Figure 1, the main hardware includes: 1. Ultrasonic sensor, with a center frequency of $500 \mathrm{KHz}$ and a relative bandwidth of $50 \%$ at $-6 \mathrm{~dB}$, the relative echo sensitivity is $-36.82 \mathrm{~dB}$, and the receiving area diameter is $15 \mathrm{~mm} ; 2$. The signal amplifier includes a preamplifier and a main amplifier, where in the bandwidth of the main amplifier (Olympus, 5662 ) is $50 \mathrm{~K}-5 \mathrm{MHz}$, there are $34 \mathrm{~dB}$ and $54 \mathrm{~dB}$ two gears; 3 , The high-definition digital oscilloscope (KEYSIGHT, DSOS054A) has a maximum sampling frequency of $20 \mathrm{GS} / \mathrm{s}$, a bandwidth of $500 \mathrm{MHz}$, four analog channels, and a built-in USB interface; 4, 3D Mobile platform (Daheng Optoelectronics, GCD-202050M), which uses a stepper motor controller (Daheng Optoelectronics, GCD-040101M). The light source is a custom diode laser with a repetition rate of $1 \mathrm{kHz}$. The output wavelength is $650 \mathrm{~nm}$, the pulse width is $1 \mu \mathrm{s}$, and the pulse energy is $1 \mu \mathrm{J} ; 5$. Personal computer.

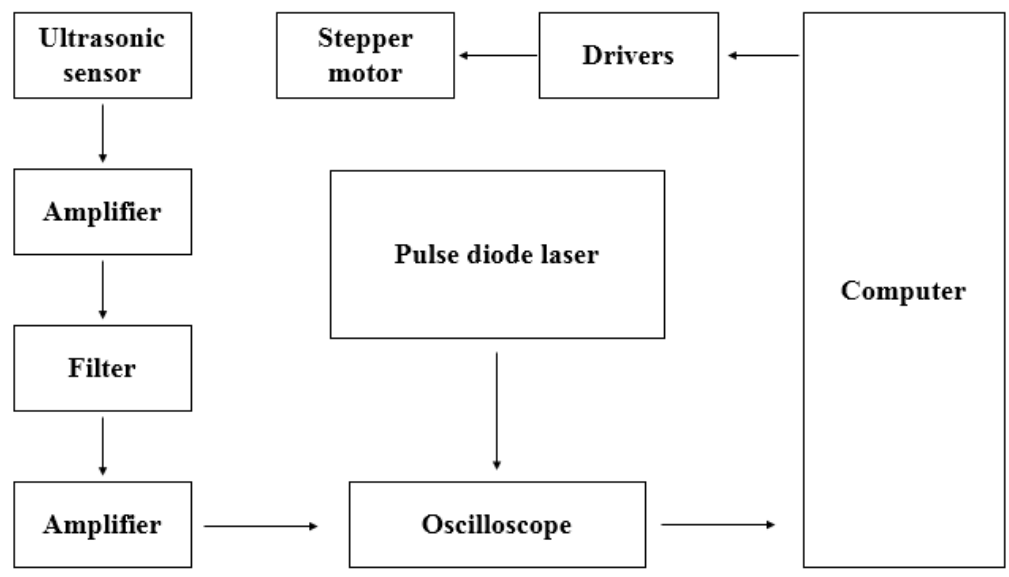

Fig. 1. Truss diagram of photoacoustic microscopy system.

The experimental device of the whole test system is shown in Figure 2. The working principle is as follows: The continuous wave laser diode is modulated into a pulse output by the drive circuit. When the long pulse laser is microscopically focused by the reflective microscope objective and irradiated on the biological tissue, the tissue absorbs the light energy to heat up together, and the temperature rise causes thermal expansion to generate a photoacoustic signal, which is transmitted to the sensor via the ultrasonic coupling fluid. After receiving the photoacoustic signal, the sensor is input to the high-definition digital oscilloscope through the pre-amplification, main amplifier and filter. The data is averaged and collected by the high-definition digital oscilloscope and transmitted to the computer by the USB interface. Finally, data processing and image reconstruction are performed using a 
program written based on the MATLAB software platform. Among them, each pulsed laser is accompanied by a synchronous triggering electrical signal, and each synchronous triggering signal is used to trigger a high-definition digital oscilloscope, which will capture the photoacoustic signal excited by each laser to the biological tissue. In the experiment, the signal acquisition of each point on the sample is called A-Line, and each A-Line signal averages 64 times, and 100 sets of data are collected in the $\mathrm{X}$-axis direction and the $\mathrm{Y}$-axis direction respectively, which constitutes a photoacoustic microscopy system. Two-dimensional data, called raster scanning. Image reconstruction of the raster scan data can obtain a two-dimensional image of biological tissue photoacoustic imaging.

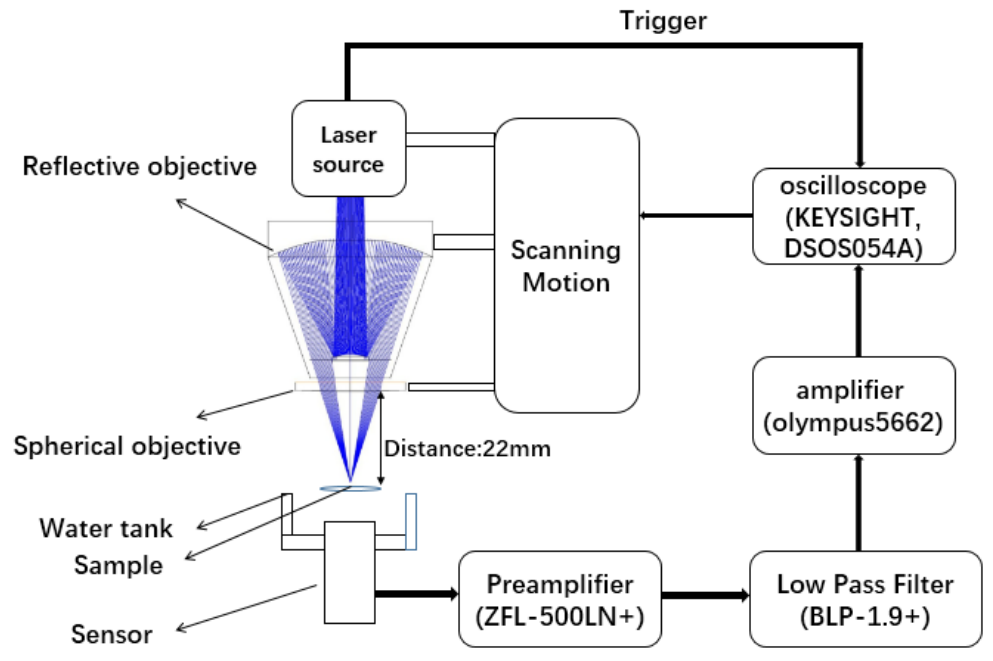

Fig. 2. Photoacoustic imaging system device.

\section{Software design}

\subsection{Photoacoustic microscope control program flow chart design}

In order to ensure the continuous operation of the electronically controlled translation stage control and realize the parameter configuration function, the core part of the main program is implemented by a "for" loop, and the main program is embedded in the sequence structure as a whole. Each time the sequence structure is completed, the data acquisition of the XY plane is completed. The "for" loop includes five states: Start, Initialize, Acquire, Data Storage, and Control Motor. The development process is shown in Figure (3).

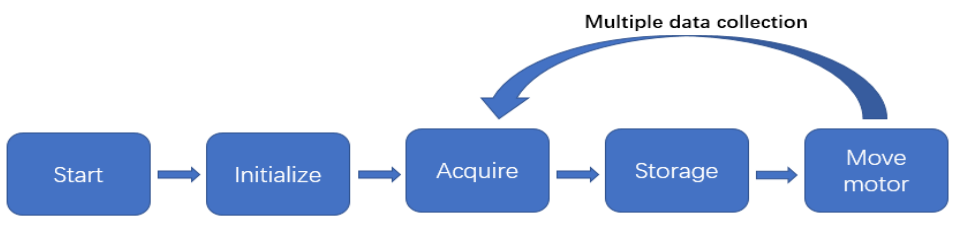

Fig. 3. Development process flow diagram of photoacoustic acquisition system. 
When the sequential structure is running, you need to click the "Start" button on the front panel to start the acquisition process. The front panel is shown in Figure (4). After starting the acquisition program, the sequence structure will execute the "Initialize" command to initialize the configuration of the oscilloscope parameters and read the serial port configuration of the electronically controlled translation stage. The next step is to execute the "Acquire" and "Storage" commands, collect and store the data in the front panel waveform graph to the PC, and then execute the "Move motor" command to control the motor movement to complete a data acquisition. Then jump to "Acquire" and repeat the command to complete the raster scanning.

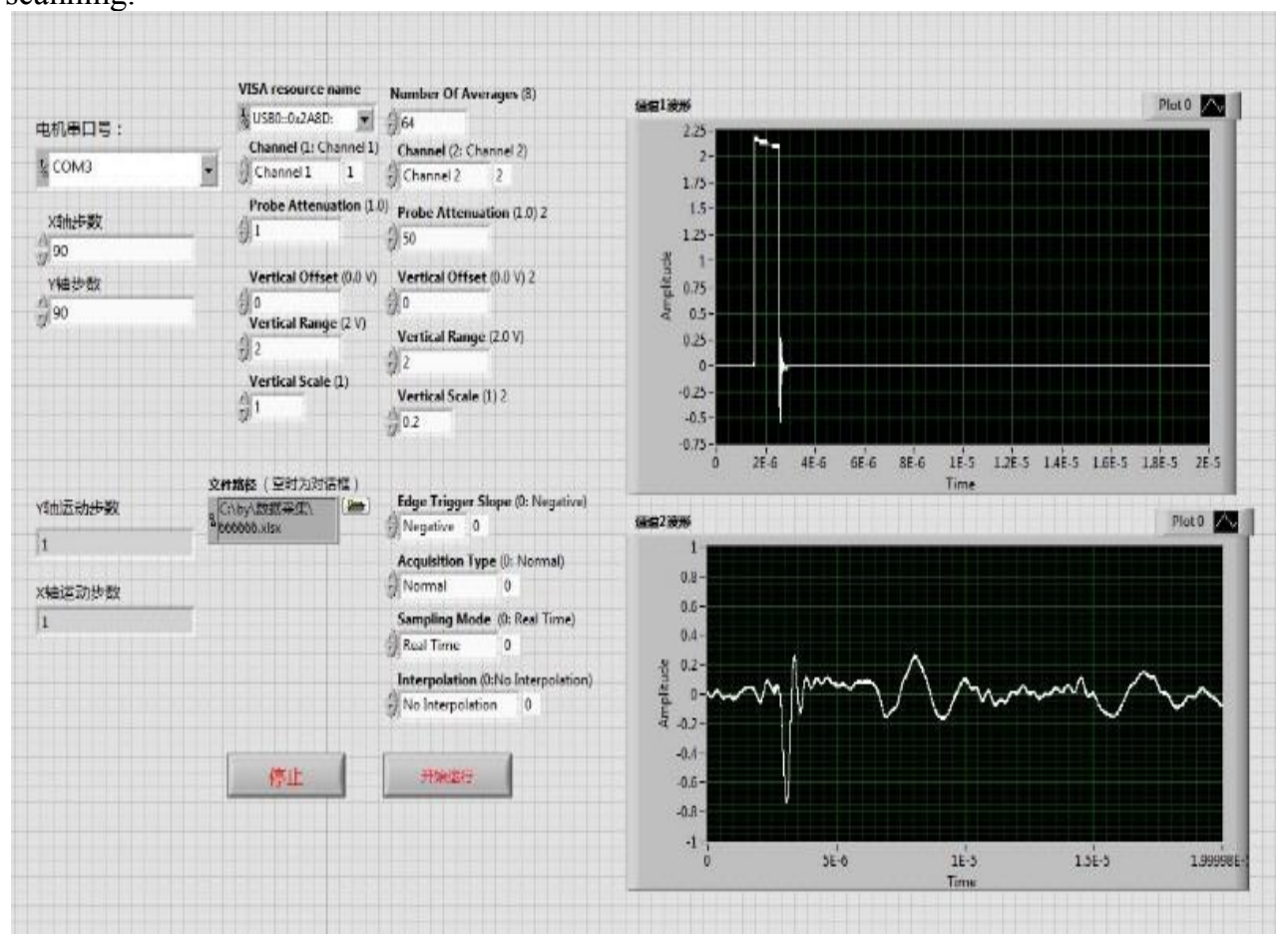

Fig. 4. Front panel photoacoustic signal diagram.

\subsection{Photoacoustic microscope control program design}

The control program of the photoacoustic microscope consists of two parts: the data acquisition program and the motor scan control program. The data acquisition program can be divided into four modules: initial setting, acquisition condition setting, data acquisition and data storage. The acquisition condition setting module is mainly for setting the parameters of the high-definition digital oscilloscope, such as selecting the oscilloscope's sampling rate to be $200 \mathrm{MS} / \mathrm{s}$, rising edge triggering, setting the signal acquisition depth, and the average number of signals. The data acquisition module is shown in Figure 5(a), which is the core of the data acquisition program. The motor scan control program needs to preset the step speed and step precision of the motor, and then control the movement of each axis of the motor in the programmed LabVIEW program. The control program of the motor is shown in Figure 5(b). 

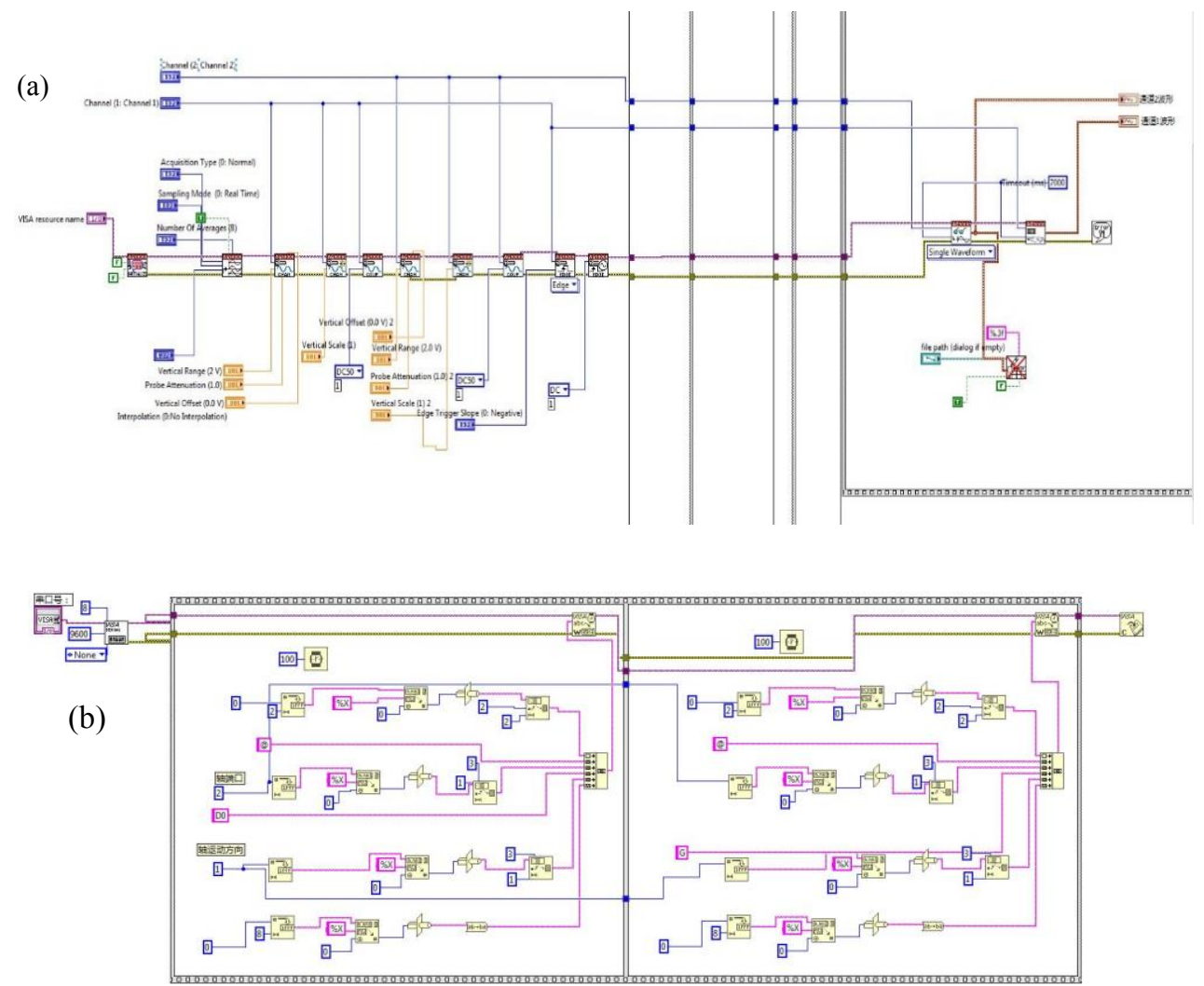

Fig. 5. (a) data acquisition module; (b) motor control program.

\section{Biological experiments}

Blood vessels are an important part of an organism, spread throughout the body, providing biological tissue with the nutrients needed to sustain life. In biological organisms, any biological tissue lesions can cause changes in vascular structure and intravascular composition. Therefore, non-invasive vascular imaging can solve many biomedical problems. And has a wide range of research and application value in the field of biomedicine.

Combined with the data acquisition module developed by LABVIEW software platform, the photoacoustic imaging test was performed on the ear blood vessels of mouse, and Fig. 5(a) is the photo of the sample. Figure 5(b) is a maximum projection of the photoacoustic microscopy of the sample with a scanned imaging area of $2 \mathrm{~mm} \times 2 \mathrm{~mm}$. In the experiment, the electronically controlled translation stage drives the laser diode source to have a scanning step of $20 \mu \mathrm{m}$ in the X-Y plane. can be seen from the experimental results that the photoacoustic reconstruction images are consistent with the distribution of mouse ear vessels, in which the main vessel and microvascular branch structures are clearly imaged, and the surrounding subcutaneous capillary network is completely reproduced with an imaging resolution of about $14 \mu \mathrm{m}$. The experiment proved the feasibility of the system to study the biological subcutaneous vascular structure and subcutaneous blood vessel distribution imaging. 


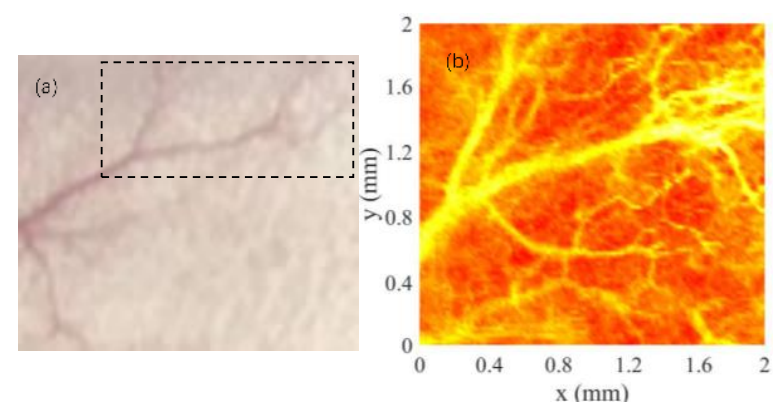

Fig. 5. Photograph of the mouse ear microvasculature and reconstructed images.

\section{Conclusion}

This paper proposes a small-scale photoacoustic microscopy system based on LabVIEW 2015 virtual instrument development platform. It uses the graphical language LabVIEW development system software to realize high-resolution mouse ear vascular structure imaging.

The next step is to combine multi-wavelength laser diode excitation, which is expected to develop into a mark-free, low-cost, miniaturized reflective photoacoustic functional microscopy technology, providing a real-time monitoring imaging and biometric identification for subcutaneous microcirculation structures

\section{References}

1. Huang Li-na, Yu Xiao-feng, Ding Zhi-hua. Numerical Analysis of Double Pass Rapid Scanning Optical Delay Line in Optical Coherence Tomography[J]Acta Photonica Sinica, 2005 (11): 1663-1665.

2. Xie Shu-sen Li Hui. Principles and techniques for measuring the optical properties of biological tissues. [J]. Chinese Journal of Biomedical Engineering, 1997, 16(4):237-332

3. Yang Zi-bin. Review and Prospect on Biomedical engineering [J]. Acta Actademiae Medicinae Sinicae, 2000, 22(2):280-210.

4. Jackson V P, Hendrick R E, Feig S A, et al. Imaging of the radiographically dense breast. [J]. Radiology, 1993, 188(2):297-301.

5. Wong T T W, Zhang R, Zhang C, et al. Label-free automated three-dimensional imaging of whole organs by microtomy-assisted photoacoustic microscopy[J]. Nature Communications, 2017, 8(1):1386.

6. Murray T W, Haltmeier M, Berer T, et al. Super-resolution photoacoustic microscopy using blind structured illumination[J]. Optica, 2017, 4(1):17-22.

7. Yao J, Wang L, Yang J M, et al. High-speed label-free functional photoacoustic microscopy of mouse brain in action[J]. Nature Methods, 2015, 12(5):407-410.

8. Yang $\mathrm{X}$, Jiang $\mathrm{B}$, Song $\mathrm{X}$, et al. Fast axial-scanning photoacoustic microscopy using tunable acoustic gradient lens[J]. Optics Express, 2017, 25(7):7349-7359.

9. Yang F, Song W, Zhang CL, et al. Broadband graphene-based photoacoustic microscopy with high sensitivity[J]. Nanoscale, 2018, 10:8606-8614

10. Yang M, Zhao L, He X, et al. Photoacoustic/ultrasound dual imaging of human thyroid cancers: An initial clinical study[J]. Biomedical Optics Express, 2017, 8(7): 3449-3457. 
11. Stylogiannis A, Prade L, Buehler A, et al. Continuous wave laser diodes enable fast optoacoustic imaging[J]. Photoacoustics, 2018, 9:31-38

12. Liang Y, Jin L, Wang L, et al. Fiber-Laser-Based Ultrasound Sensor for Photoacoustic Imaging[J]. Scientific Reports, 2017, 7:40849

13. Wang T, Nandy S, Salehi H S, et al. A low-cost photoacoustic microscopy system with a laser diode excitation[J]. Biomedical Optics Express, 2014, 5(9):3053-3058.

14. Daoudi K, Van d B P J, Rabot O, et al. Handheld probe integrating laser diode and ultrasound transducer array for ultrasound/photoacoustic dual modality imaging[J]. Optics Express, 2014, 22(21): 26365-26374.

15. Wang P H, Li M L. DVD pickup head based optical resolution photoacoustic microscopy $[\mathrm{J}]$. Proceedings of SPIE - The International Society for Optical Engineering, 2012, 8223:78.

16. Erfanzadeh M, Kumavor P D, Zhu Q. Laser scanning laser diode photoacoustic microscopy system[J]. Photoacoustics, 2018, 9:1-9.

17. Beckmann M F, Schwab H M, Schmitz G. Optimized SNR simultaneous multispectral photoacoustic imaging with laser diodes[J]. Optics Express, 2015, 23(2):1816-1828.

18. Zeng L, Liu G, Yang D, et al. Cost-efficient laser-diode-induced optical-resolution photoacoustic microscopy for two-dimensional/three-dimensional biomedical imaging[J]. Journal of Biomedical Optics, 2014, 19(7):076017. 